Check for updates

Cite this: RSC Adv., 2017, 7, 29386

Received 25th January 2017

Accepted 23rd May 2017

DOI: $10.1039 / c 7 r a 01145 h$

rsc.li/rsc-advances

\section{The chain order of binary unsaturated lipid bilayers modulated by aromatic-residue-containing peptides: an ATR-FTIR spectroscopy study $\dagger$}

\begin{abstract}
Bo Peng, (D) Xiao-Yan Ding, Chao Sun, Ya-Nan Yang, Yu-Jiao Gao and Xin Zhao*
In this study, we described the chain order change of binary unsaturated lipid bilayers by studying pure 2oleoyl-1-pamlitoyl-sn-glyecro-3-phosphocholine (POPC) bilayers, 2-oleoyl-1-pamlitoyl-sn-glyecro-3glycerol (POPG) bilayers, and POPC/POPG bilayers. The interactions between the POPC/POPG membrane and three model peptides containing basic, aromatic, and hydrophobic residues were studied to investigate the effects of the peptide orientation on the acyl chain order of the POPC/POPG bilayers. Attenuated total reflectance-Fourier transform infrared (ATR-FTIR) spectroscopic studies showed that the acyl chain order of the POPC/POPG bilayers decreased as compared to that of the pure POPC or POPG bilayers. The chain order of the POPC/POPG bilayers significantly decreased when Gravin was added as compared to that when Vamp2 or Antp was added. Fluorescence spectroscopic measurements further show that the tryptophan of the three peptides may interact with the hydrophobic region of the POPC/POPG bilayers, thus resulting in a change in the conformational order of the POPC/POPG acyl chains. This study may provide valuable insight into the dynamics and flexibility of lipid bilayers with different lipids and highlights the importance of aromatic residues in peptide binding to the membrane.
\end{abstract}

\section{Introduction}

Biological membranes display a complex variety of different lipids. Variations in the lipid composition ${ }^{\mathbf{1}}$ and the size of lipid bilayer aggregates ${ }^{2}$ are well-known to significantly affect membrane properties, especially the lipid composition. Different lipid compositions make the physicochemical properties and physical behavior, including the permeability, ${ }^{3}$ melting transition behavior, ${ }^{4}$ fluidity, curvature, thickness, and charge distribution, ${ }^{5}$ of the membranes complex. In addition, the lipid composition is also involved in the insertion and stabilization of amino acids in the membranes ${ }^{6}$ and the structure and function of the membrane proteins. ${ }^{7}$

The lipid composition is distinguished by different head groups, acyl chain lengths, and acyl chain unsaturation. The most common phosphatidylcholine (PC) molecules are present in all mammalian cell membranes except for those in the brain. ${ }^{8}$ Phosphatidylglycerol (PG) is the only major phospholipid in the thylakoid membrane of plant chloroplasts and cyanobacteria. ${ }^{9}$ Both POPG and POPC contain an unsaturated (C18) and a saturated (C16) fatty acid as the lipophilic tails.

Shanghai Key Laboratory of Magnetic Resonance, Department of Physics, School of Physics and Materials Science, East China Normal University, Shanghai 200062, P. R. China. E-mail: xzhao@phy.ecnu.edu.cn; Fax: +86 21 62234329; Tel: +86 21 62234329

$\uparrow$ Electronic supplementary information (ESI) available. See DOI: 10.1039/c7ra01145h
While the hydrophilic head group of POPC is zwitterionic, the head group attached to the diacylglycerol backbone in POPG is negatively charged.

Many peripheral proteins, such as myristoylated alanine-rich C kinase substrate (MARCKS), ${ }^{\mathbf{1 0}}$ FAT C-terminal (FATC) domain, ${ }^{11}$ and LL37, ${ }^{12}$ contain clusters of basic and aromatic residues that interact with lipid bilayers. Generally, aromatic and basic residue motifs are important for the anchoring of proteins to the biomembranes, ${ }^{13}$ particularly aromatic residues, which may play key roles as efficient anchors during binding at the phospholipid membrane interface. ${ }^{\mathbf{1 4 1 5}}$ The interactions between aromatic residue-containing peptides and membrane bilayers with different lipid compositions are complex. Using neutron reflectivity (NR), electron spin resonance (ESR), fluorescence titration, and molecular dynamics (MD) simulations, Vitiello et al. showed that the lipid composition modulated the interaction of the three tryptophan-containing octapeptides (C8) with membrane bilayers and the C8 may interact with the cholesterol-interacting sphingomyelin and induce a significant reduction of the POPC acyl chain order. ${ }^{16}$ Sanders et al. reported that the tryptophan-rich wild type (Pin-b) and mutant (Trp44 to Arg44 mutant, Pin-bs) puroindoline proteins interacted with mixed phosphatidylethanolamine (PE) and PG with different lipid selectivity. Moreover, using FTIR spectroscopy, surface pressure measurements, and Brewster angle microscopy, it was found that Pin-b could insert into the head groups of the mixed PE and PG more deeply than Pin-bs. ${ }^{17}$ Using dual polarisation interferometry (DPI), Hirst et al. demonstrated that the HPA3 
peptide, which contains basic and aromatic residues, may bind to different lipid bilayers and induce unsaturated bilayers to be more disordered than saturated bilayers. ${ }^{18}$ It has been reported that the interactions between C6-LfB6 peptide, which contains basic and aromatic residues, and POPE/POPG or POPC bilayers depend on the lipid composition. C6-LfB6 may bind to the negatively charged POPE/POPG bilayer interface and cause no change in the lipid acyl chain order, whereas for zwitterionic POPC bilayers, it can more deeply insert into the POPC membrane and induce a slight decrease in the order of the lipid acyl chains, as observed via solid-state ${ }^{2} \mathrm{H}$ and ${ }^{31} \mathrm{P}$ nuclear magnetic resonance (NMR) and all-atom MD simulations. ${ }^{19}$ In addition, the arginine-rich RL9 and tryptophan-containing RW9 peptides may locate at the interface of POPC, POPG, and POPC/ POPG membranes and water, where RW9 has a larger influence in increasing membrane flexibility than RL9 based on isothermal titration calorimetry (ITC), solid-state NMR, and MD simulations. ${ }^{20}$ However, the perturbation of aromatic residuecontaining peptides interacting with binary unsaturated lipid bilayers remains unclear, and studies to better understand this possible effect associated with the lipid composition are of particular importance.

Fourier transform infrared (FTIR) spectroscopy, a common non-invasive biophysical approach, has been broadly used to selectively monitor the perturbations on the polar head groups, the interfacial region, and the hydrophobic acyl chains of lipids. ${ }^{21,22}$ It provides valuable information about biomembranes: the phase transition and order-disorder state of lipid membranes, ${ }^{23}$ the asymmetry and assemblies of bilayers, ${ }^{24}$ the structural changes in membranes induced via interactions with small molecules, ${ }^{25,26}$ or antimicrobial cationic lipopeptides, ${ }^{27}$ etc. Changes in the width and position of the lipid IR peaks can provide useful information regarding lipid membrane dynamics ${ }^{28}$ and interactions of lipids with proteins. ${ }^{29}$ In the current study, we aimed at investigating the chain order of binary unsaturated lipid mixtures and the perturbation of binary unsaturated lipids with aromatic residue-containing peptide binding. We used polarised ATRFTIR spectroscopy to investigate the acyl chain order of the mixed POPC/POPG bilayers and the perturbation of the chain order in the POPC/POPG membrane bilayers modulated by three specially designed model peptides. In addition, the possible role of the Trp residues in the process of peptide interaction with POPC/POPG bilayers was qualitatively analysed via fluorescence spectroscopy.

\section{Methodology}

\subsection{Materials}

POPC and POPG were obtained as powders from Avanti Polar Lipids without further purification. $\mathrm{D}_{2} \mathrm{O}(99.9 \%)$ was obtained from Cambridge Isotope Laboratory. Trp-OMe, Vamp2 (83-95) KLKRKYWWKNLKM, Antp (43-58) RQILIWFQNRRMKWKK, and Gravin (297-317) KKFFTQGWAGWRKKTSFRKPK were purchased from Ji'er Biochemical (Shanghai) Co. Ltd. The molecular formulae of POPC and POPG and the sequences of model peptides are shown in Fig. 1.
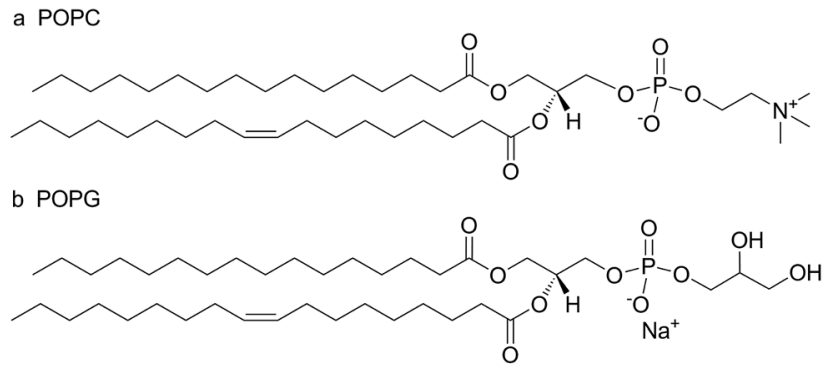

c Vamp2(83-95) $\underline{K} L \underline{K R K} Y W W \underline{K N L \underline{K M}}$

d Antp(43-58) $\underline{R} Q / L I W F Q N \underline{R R M} \underline{K} W \underline{K K}$

e Gravin(297-317) KKFFTQGWAGW $\underline{\text { RKKTSFRKPK }}$

Fig. 1 Molecular formulae of a zwitterionic lipid (a) POPC (2-oleoyl-1pamlitoyl-sn-glyecro-3-phosphocholine), an anionic lipid (b) POPG (2-oleoyl-1-pamlitoyl-sn-glyecro-3-glycerol) (sodium salt), sequences of (c) Vamp2 (83-95), (d) Antp (43-58), and (e) Gravin (297-317). Basic residues (lysine and arginine) have been underlined. Aromatic residues (phenylalanine, tryptophan, and tyrosine) are presented in bold. Hydrophobic residues (leucine, isoleucine, glycine, alanine, proline, phenylalanine, tryptophan, and tyrosine) are presented in italics.

\subsection{Preparation of the multilamellar vesicles (MLVS)}

Lipids were dissolved in a mixture of chloroform and methanol $(2: 1)$, and then, the solvent was evaporated under a stream of nitrogen gas and lyophilised for several hours. Lipids were then dissolved in cyclohexane, evaporated under a flow of nitrogen gas, and then desiccated under vacuum to remove any residual solvent. Dried films were hydrated with $20 \mathrm{mM}$ 4-(2hydroxyethyl)-1-piperazineethanesulphonic acid (HEPES) and $30 \mathrm{mM}$ sodium chloride ( $\mathrm{NaCl}$ ) buffer ( $\mathrm{pH} 7.0$ ), subjected to 10 cycles of freeze-thaw-sonication, and then kept under vacuum for several hours. Dried lipid films were hydrated with $\mathrm{D}_{2} \mathrm{O}$ to $50 \%$ water by mass. Then, the lipid samples were incubated at $37{ }^{\circ} \mathrm{C}$ for 2 days. The POPC/POPG molar ratio was $10: 3$. The model peptides were added to the POPC/POPG MLVs prior to lyophilisation at a molar ratio of $1: 50$. The molar concentration of the peptides was about $2 \%$.

\subsection{ATR-FTIR spectroscopy}

The ATR-FTIR experiments were performed via a Nicolet 8700 USB IR spectrometer equipped with a deuterated triglycine sulphate (DTGS) detector. A standard PIKE Technologies ATRMax device equipped with a ZnSe grating polariser that can be oriented parallel or perpendicular to the ATR plane was used. The internal reflection element was a $56 \mathrm{~mm} \times 10 \mathrm{~mm} \times 4 \mathrm{~mm}$ ZnSe plate with an aperture angle of $45^{\circ}$ yielding 7 internal reflections. A schematic of the ATR device with an internal ZnSe reflection plate covered with the POPC bilayers, POPC/POPG bilayers, and POPC/POPG bilayer-peptide complexes is shown in Fig. 2. Spectra were obtained with $4 \mathrm{~cm}^{-1}$ resolution between 650 and $4000 \mathrm{~cm}^{-1}$ region at $298 \mathrm{~K}$, and 128 scans were obtained for each spectrum. The dichroic ratio $\left(R^{\mathrm{ATR}}\right)$ values are the average values obtained from three measurements. The orientation order parameter $f$ and the average angle of the lipid 


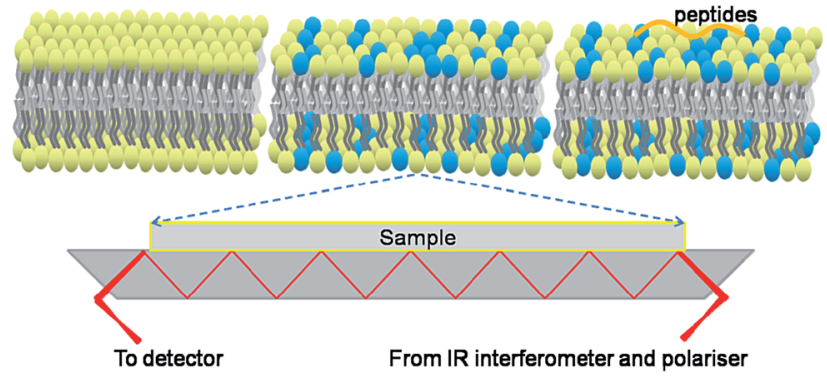

Fig. 2 A schematic of the ATR device with an internal ZnSe reflection plate covered with the POPC bilayers, POPC/POPG bilayers, and POPC/POPG bilayer-peptide complexes (yellow: POPC and blue: POPG).

chains relative to the membrane normal $\gamma$ were calculated following a previous method. ${ }^{30,31}$ The tilt angle $\beta$ of the $\alpha$-helical component from the membrane normal was calculated via a previous method..$^{32-35}$ Detailed information about the polarised ATR-FTIR data analysis is shown in the ESI. $\dagger$ All the experiments were analysed via an OMNIC 8.1 software (Thermo Fisher Scientific Inc.). Second derivative spectra were obtained via a 5-point Norris derivative method.

\subsection{Fluorescence spectroscopy}

Fluorescence emission spectra were obtained via a Horiba JobinYvon Fluoromax-4 spectrofluorometer. The bandwidth for emission was $4 \mathrm{~nm}$, and an excitation wavelength of $295 \mathrm{~nm}$ was used to ensure that light was almost entirely absorbed by the tryptophanyl groups. ${ }^{36}$ All spectra were obtained at room temperature.

\section{Results and discussion}

\subsection{FTIR spectra of the POPC bilayers, POPG bilayers, and POPC/POPG bilayers}

Fig. 3A and $\mathrm{C}$ show the FTIR spectra of the POPC bilayers, POPG bilayers, and POPC/POPG bilayers in the $3050-2800 \mathrm{~cm}^{-1}$ and 1800-1000 $\mathrm{cm}^{-1}$ regions, respectively. The important vibrational peaks could be clearly assigned. ${ }^{37}$ For the POPC bilayers, the band of the $=\mathrm{CH}$ stretching mode at $3010.2 \mathrm{~cm}^{-1}$ and the $\mathrm{C}=\mathrm{C}$ stretching mode at $1651.4 \mathrm{~cm}^{-1}$ can be taken into consideration. The $\mathrm{CH}_{2}$ antisymmetric stretching band at $2919.3 \mathrm{~cm}^{-1}$ and $\mathrm{CH}_{2}$ symmetric stretching band at 2850.3 $\mathrm{cm}^{-1}$ were also unambiguously identified. In addition, the $\mathrm{PO}_{2}{ }^{-}$ antisymmetric stretching band at $1240.5 \mathrm{~cm}^{-1}$, the $\mathrm{PO}_{2}{ }^{-}$ symmetric stretching band at $1091.3 \mathrm{~cm}^{-1}$, and the $\mathrm{C}-\mathrm{N}^{+}-\mathrm{C}$ symmetric stretching band at $919.4 \mathrm{~cm}^{-1}$ were also taken into consideration. For the POPG bilayers, the band of the $=\mathrm{CH}$ stretching mode at $3010.0 \mathrm{~cm}^{-1}$, the $\mathrm{C}=\mathrm{C}$ stretching mode at $1651.8 \mathrm{~cm}^{-1}$, the $\mathrm{CH}_{2}$ antisymmetric stretching band at 2920.6 $\mathrm{cm}^{-1}$, and the $\mathrm{CH}_{2}$ symmetric stretching band at $2850.7 \mathrm{~cm}^{-1}$ can be clearly assigned. These bands are similar to those for the POPC bilayers. While the $\mathrm{PO}_{2}{ }^{-}$antisymmetric stretching band at $1220.0 \mathrm{~cm}^{-1}$ and the $\mathrm{PO}_{2}{ }^{-}$symmetric stretching band at $1094.6 \mathrm{~cm}^{-1}$ were also taken into consideration, these bands were different from those for the POPC bilayers.
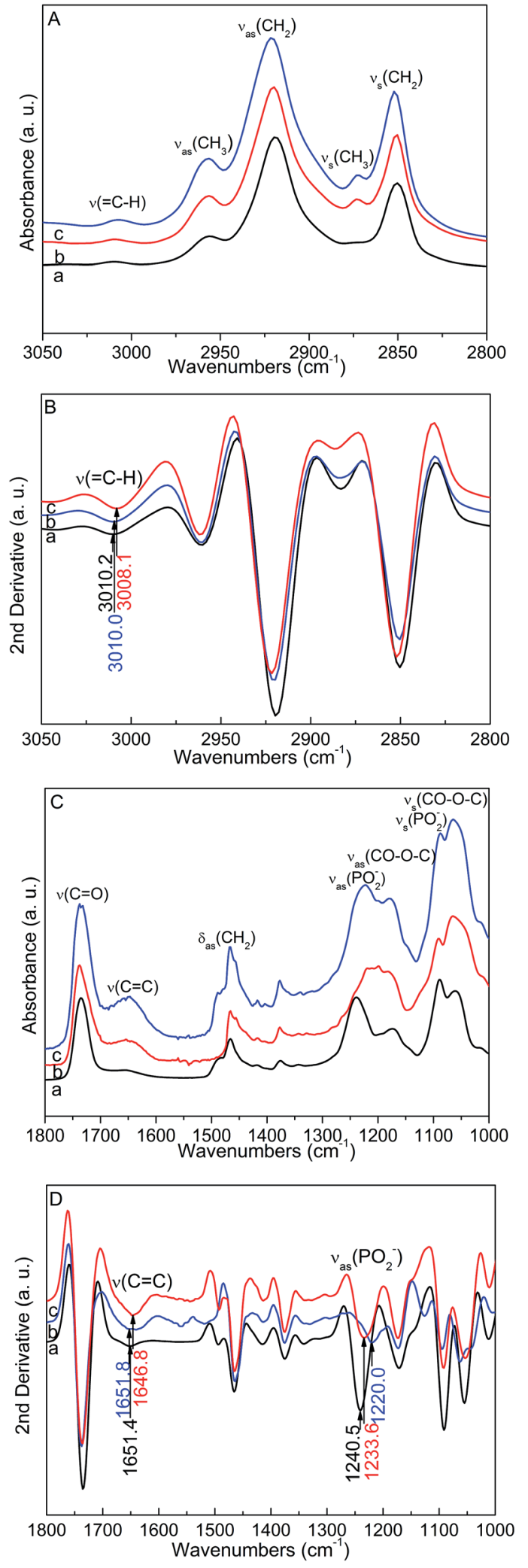

Fig. 3 FTIR spectra of (a) POPC bilayers, (b) POPG bilayers, and (c) POPC/POPG bilayers. (A) FTIR spectra in the $3050-2800 \mathrm{~cm}^{-1}$ region. (B) Second-derivative spectra in the $3050-2800 \mathrm{~cm}^{-1}$ region. (C) FTIR spectra in the $1800-1000 \mathrm{~cm}^{-1}$ region. (D) Second-derivative spectra in the $1800-1000 \mathrm{~cm}^{-1}$ region. 
For the POPC/POPG bilayers, the vibrational frequencies of the $=\mathrm{CH}$ and $\mathrm{C}=\mathrm{C}$ stretching modes showed red shifts. The second-derivative FTIR spectra of the POPC, POPG, and POPC/ POPG bilayers in the $3050-2800 \mathrm{~cm}^{-1}$ and $1800-1000 \mathrm{~cm}^{-1}$ regions are presented in Fig. 3B and D, respectively. The band of the $=\mathrm{CH}$ stretching mode locates at $3008.1 \mathrm{~cm}^{-1}$ and the $\mathrm{C}=\mathrm{C}$ stretching mode locates at $1646.8 \mathrm{~cm}^{-1}$ in the POPC/POPG bilayers. Compared to the POPC bilayers, the $=\mathrm{CH}$ and $\mathrm{C}=\mathrm{C}$ stretching modes show red shifts of $2.1 \mathrm{~cm}^{-1}$ and $4.6 \mathrm{~cm}^{-1}$, respectively, in the mixed POPC/POPG bilayers. Moreover, the vibrational frequencies of the $\mathrm{CH}_{2}$ antisymmetric and symmetric stretching modes show blue shifts of $2.5 \mathrm{~cm}^{-1}$ and $1.6 \mathrm{~cm}^{-1}$, respectively. Moreover, the frequency, width, and intensity of the antisymmetric and symmetric $\mathrm{CH}_{2}$ and $\mathrm{CH}_{3}$ stretching bands can be used to monitor the order state of the membranes. ${ }^{38}$ These results demonstrate that the order of the acyl chains decreases in the POPC/POPG bilayers as compared to that in the pure POPC bilayers. In addition, the $\mathrm{PO}_{2}{ }^{-}$antisymmetric stretching band at $1233.6 \mathrm{~cm}^{-1}$ shows a red shift of $6.9 \mathrm{~cm}^{-1}$ and the $\mathrm{C}-\mathrm{N}^{+}-\mathrm{C}$ antisymmetric stretching band at $922.4 \mathrm{~cm}^{-1}$ shows a small blue shift of $3 \mathrm{~cm}^{-1}$ for the POPC/ POPG bilayers as compared to those for the POPC bilayers. These bands are mainly associated with the head groups of lipids. The lipid headgroup packing depends on the structure of the headgroup as well as on the fatty acyl chain structure. ${ }^{5}$ Our FTIR results demonstrate that the acyl chain order in the case of a binary lipid system composed of POPC and POPG decreases to some extent although based on MD simulations, Janosi et al. have reported that the POPC bilayers containing $\sim 23 \%$ POPG differ from their neutral counterparts only at the headgroup. ${ }^{39}$ This discrepancy may be caused by the differences in the chosen force field for the MD simulations. The head group of POPC is zwitterionic, whereas the head group of POPG is negatively charged. With the different head groups in the POPC/POPG bilayers, the packing and arrangements in the polar head group and interfacial regions of the lipid matrix are different from those of the pure POPC or POPG bilayers. Thus, we can speculate that the acyl chain order of the POPC/POPG bilayers decreases to some extent as compared to that of the POPC or POPG bilayers.

\subsection{FTIR spectra of the POPC/POPG bilayers and POPC/ POPG bilayer-model peptide complexes}

We used several model peptides to further investigate the chain order of the mixed POPC/POPG bilayers. Initial spectra of the POPC/POPG bilayers were obtained as references. Fig. 4A shows the FTIR spectra of the POPC/POPG bilayers and POPC/POPG bilayer-model peptide complexes in the $3050-2800 \mathrm{~cm}^{-1}$ region. The important vibrational peaks could also be clearly assigned. ${ }^{37}$ In the second-derivative FTIR spectra in the 3050$2800 \mathrm{~cm}^{-1}$ region of the POPC/POPG bilayers and complexes of model peptides with the POPC/POPG bilayers (Fig. 4B), a red shift of $1.2 \mathrm{~cm}^{-1}$ for the $=\mathrm{CH}$ stretching band of the POPC/ POPG bilayers was observed when Gravin was added. However, there were negligible changes in the frequency of the $=\mathrm{C}-\mathrm{H}$ stretching band when Antp (a red shift of about 1.1
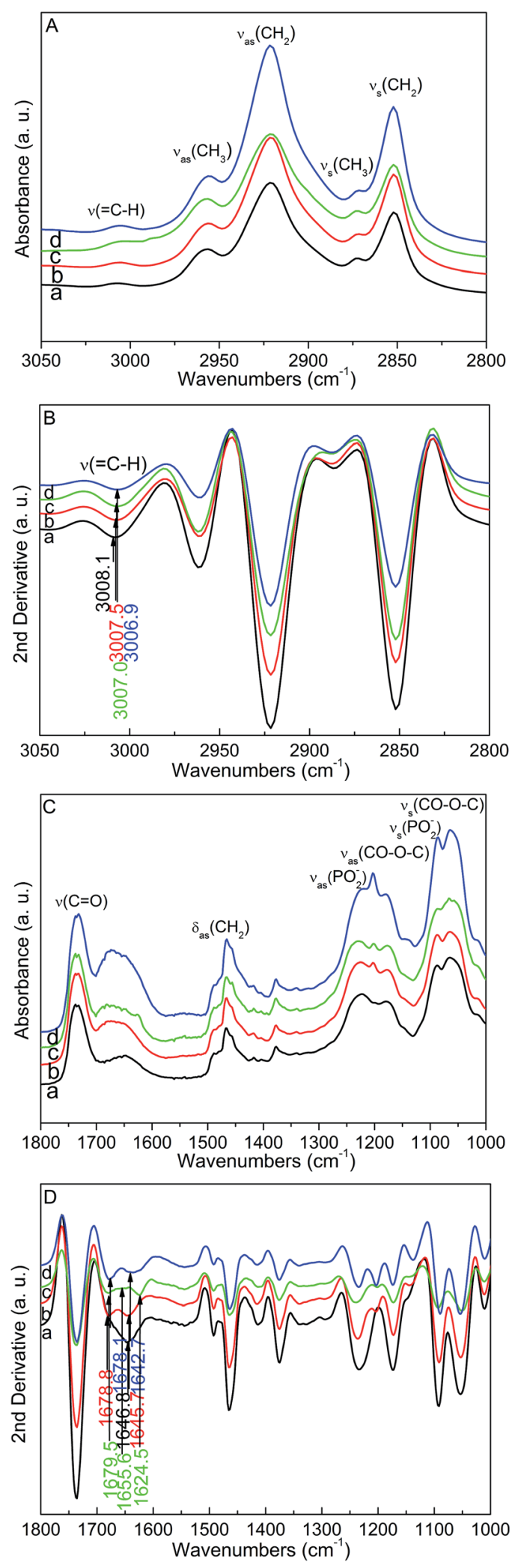

Fig. 4 FTIR spectra of the POPC/POPG bilayers and POPC/POPG bilayer-peptide complexes. (A) FTIR spectra in the $3050-2800 \mathrm{~cm}^{-1}$ region. (B) Second-derivative spectra in the $3050-2800 \mathrm{~cm}^{-1}$ region. (C) FTIR spectra in the $1800-1000 \mathrm{~cm}^{-1}$ region. (D) Second-derivative spectra in the $1800-1000 \mathrm{~cm}^{-1}$ region. (a) POPC/POPG bilayers, (b) Vamp2 peptide with POPC/POPG bilayers, (c) Antp peptide with POPC/ POPG bilayers, and (d) Gravin peptide with POPC/POPG bilayers. 
$\mathrm{cm}^{-1}$ ) and Vamp2 (about $0.6 \mathrm{~cm}^{-1}$ ) were added. The $\mathrm{C}=\mathrm{O}$ stretching mode of ester carbonyl at $1736.4 \mathrm{~cm}^{-1}$ was mainly associated with the POPC/POPG bilayers. Fig. $4 \mathrm{C}$ shows that the shoulder peaks near the $\nu(\mathrm{C}=\mathrm{O})$ band became widest when Gravin was added. In addition, shoulder peaks near the $\nu(\mathrm{C}=\mathrm{O})$ were observed when Antp and Vamp2 were added. These peaks are associated with the amide I band and amino acid side chains in this region when model peptides are mixed into binary lipid bilayers. The second-derivative spectra in the 1800$1000 \mathrm{~cm}^{-1}$ region (Fig. 4D) show that main shoulder peaks appeared at $1679.5 \mathrm{~cm}^{-1}, 1655.6 \mathrm{~cm}^{-1}$, and $1624.5 \mathrm{~cm}^{-1}$ for the Antp-POPC/POPG bilayer complex. These may be associated with the $\gamma$-turn, the anti-parallel $\beta$-sheet, the $\alpha$-helix, or the aggregated $\beta$-sheet. ${ }^{40}$ For the Vamp2-POPC/POPG bilayer complex, main shoulder peaks appeared at $1678.8 \mathrm{~cm}^{-1}$ and $1645.7 \mathrm{~cm}^{-1}$. These may be associated with the anti-parallel $\beta$ sheet, the $\gamma$-turn, or the $\alpha$-helix. ${ }^{40}$ They also appeared at 1678.1 $\mathrm{cm}^{-1}$ and $1642.7 \mathrm{~cm}^{-1}$ in the Gravin-POPC/POPG bilayer complex and these may be associated with the anti-parallel $\beta$ sheet, the $\gamma$-turn, or the $\alpha$-helix. ${ }^{40}$ As the FTIR absorption bands of this region are complex, variations in the frequency of the $\mathrm{C}=\mathrm{C}$ stretching band of the POPC/POPG acyl chains have not yet been distinguished and analysed. Additionally, the shape of the $\mathrm{PO}_{2}^{-}$antisymmetric stretching band shows variation to some extent when the three peptides are mixed into the POPC/ POPG bilayers. These phenomena are mainly due to the interactions between the $\mathrm{PO}_{2}{ }^{-}$groups of the POPC/POPG bilayers and the peptides.

Using ATR-FTIR spectroscopy, information on the secondary structures and orientations of peptides can be readily obtained. ${ }^{41-43}$ Table 1 shows the parameters for s- and p-polarized ATR-FTIR spectra of the POPC/POPG bilayer-peptide complexes. According to the strength ratio of the $\mathrm{s}$ - and $\mathrm{p}$ polarized spectra, the tilt angle of the $\alpha$-helical component from the membrane normal was calculated to be $69.0^{\circ}$ in the Vamp2-POPC/POPG bilayer complex; it was calculated to be $53.6^{\circ}$ in the Antp-POPC/POPG bilayer complex; and it was determined to be $42.4^{\circ}$ in the Gravin-POPC/POPG bilayer complex.

As shown in Fig. 1, the three model peptides have different amino acid sequence motifs. All three peptides have various basic, aromatic, and hydrophobic residues in the sequence. Vamp2 (83-95) is a 13-residue peptide that contains three aromatic residues (2-Trp and 1-Tyr), six basic residues (5-Lys and 1-Arg), and two hydrophobic residues (2-Leu). Gravin (297-
$317)$ is a 21-residue peptide that contains five aromatic residues (3-Phe and 2-Trp), eight basic residues (6-Lys and 2-Arg), and four hydrophobic residues (2-Gly, 1-Ala, and 1-Pro). Antp (4358 ) is a 16-residue peptide that contains three aromatic residues (2-Trp and 1-Phe), six basic residues (3-Lys and 3-Arg), and three hydrophobic residues (2-Ile and 1-Leu). As the positively charged basic amino acid residues may generally interact with the negatively charged headgroups of lipids via electrostatic effects, ${ }^{\mathbf{4}}$ the side-chains of the arginine residues may interact with the hydrophilic moieties, such as phosphate moieties, of lipids. ${ }^{45}$ The side chain groups of basic amino acids, such as lysine, can also provide a flexible hydrocarbon spacer and enable hydrophobic interactions. ${ }^{46}$ The three peptides may adopt various orientations via interacting with the membrane. Several studies have reported that the Antp (43-58) peptide is helical at low ratios of peptide to lipid and becomes less helical at high ratios of peptide to lipid..$^{\mathbf{4}, 48}$ Gravin has the smallest tilt angle of the $\alpha$-helical component with respect to the membrane normal. It may induce the most significant conformational change and disorder of the POPC/POPG acyl chains in the membrane.

As above mentioned, we focused on the changes in the $=\mathrm{CH}$ stretching mode of the POPC/POPG bilayers. The red shifts in the frequency of the $=\mathrm{CH}$ stretching mode are found not only in POPC/POPG bilayers but also in the complexes of the model peptides with the POPC/POPG bilayers. Among the three POPC/ POPG bilayer-model peptide complexes, the maximum red shift in the frequency of the $=\mathrm{CH}$ stretching mode of the POPC/ POPG acyl chains was observed when Gravin was added. These results indicate that the acyl chain order of the binary unsaturated POPC/POPG bilayers decreases as compared to that of the pure POPC or POPG bilayers, and the chain order of POPC/POPG bilayers also decreases to some extent when the three model peptides are mixed in, especially Gravin. Therefore, we can speculate that the red shift of the $=\mathrm{CH}$ stretching band of the POPC/POPG bilayers mainly arises from the packing and conformational changes of the POPC/POPG acyl chains in the membranes. This may occur via aromatic residue-containing peptides interacting with the interface region of the POPC/ POPG bilayers and disrupting acyl chain packing and cooperativity in the POPC/POPG bilayers. The three peptides influence the structural properties of the POPC/POPG bilayers, particularly the binary lipid acyl chain packing and membrane fluidity. The amplitude of the disorder in the acyl chains is mainly

Table 1 ATR parameters for s- and p-polarized ATR-FTIR spectra of the POPC/POPG bilayer-peptide complexes

\begin{tabular}{|c|c|c|c|c|}
\hline Sample & Freq. $\left(\mathrm{cm}^{-1}\right)$ & Assignment & $A$ (s-polarized) & $A(\mathrm{p}-$ polarized $)$ \\
\hline \multirow[t]{2}{*}{ POPC/POPG and Vamp2 } & 1678.8 & $\gamma$-turn, anti-parallel $\beta$-sheet & 0.138 & 0.209 \\
\hline & 1645.7 & $\alpha$-helix & 0.128 & 0.207 \\
\hline & 1655.6 & $\alpha$-helix & 0.075 & 0.153 \\
\hline & 1624.5 & aggregated $\beta$-sheet & 0.075 & 0.135 \\
\hline POPC/POPG and Gravin & 1678.1 & $\gamma$-turn, anti-parallel $\beta$-sheet & 0.138 & 0.294 \\
\hline
\end{tabular}


determined by the sequence, structural context, and orientation of the interacting peptides.

\subsection{Dichroic properties of the POPC bilayers, POPG bilayers, POPC/POPG bilayers, and POPC/POPG bilayer-model peptide complexes}

The $\mathrm{CH} / \mathrm{CD}$ stretching vibrational and $\mathrm{CH}_{2}$ deformation modes are very useful in determining the lipid order. ${ }^{21} R^{\mathrm{ATR}}$ parameters for the POPC bilayers, POPG bilayers, POPC/POPG bilayers, and POPC/POPG bilayer-peptide complexes are shown in Table 2. The pure POPC bilayers are well-ordered with the orientation factor $f=0.40$, and the ATR parameters of the POPG bilayers are the same as those of the POPC bilayers. However, the $R^{\mathrm{ATR}}$ of lipids significantly increased in the POPC/POPG bilayers as compared to that in the pure POPC or POPG bilayers. Correspondingly, the orientation factor $f$ of the POPC/POPG bilayers decreased to $0.35 \pm 0.02$ and $\gamma$ increased to $41.2^{\circ} \pm 0.8^{\circ}$. This result further indicates that the addition of POPG induces an obvious decrease in the order of POPC acyl chains. The conformational order of the binary POPC/POPG acyl chains significantly decreases as compared to that of the single composition system.

In the POPC/POPG bilayers, the $R^{\mathrm{ATR}}$ of the acyl chains was $1.27 \pm 0.03$, the orientation factor $f$ was $0.35 \pm 0.02$, and the corresponding $\gamma$ was $41.2^{\circ} \pm 0.8^{\circ}$. The values of $R^{\mathrm{ATR}}, f$, and $\gamma$ changed to varying degrees when the three peptides were added into the POPC/POPG bilayers. The value of $R^{\mathrm{ATR}}$ considerably increased when Gravin was added, whereas only slightly increased when Vamp2 or Antp was added. The corresponding orientation factor $f$ of the POPC/POPG bilayers decreased to 0.20 \pm 0.02 , and $\gamma$ increased to $46.8^{\circ} \pm 0.8^{\circ}$ when Gravin was added. This result suggests that Vamp2 and Antp have less effect on the $\mathrm{CH}_{2}$ stretching mode of the acyl chains than Gravin. Gravin, which has a smaller tilt angle than Vamp2 and Antp, caused a significant increase in the dichroic ratio and a significant decrease in the orientation factor $f$ of the POPC/POPG acyl chains. Thus, the conformational order of the POPC/POPG acyl chains significantly decreased. These results are consistent with the results of the ATR-FTIR spectroscopy.

Table 2 ATR parameters for the POPC bilayers, POPG bilayers, POPC/ POPG bilayers, and POPC/POPG bilayer-peptide complexes ${ }^{a}$

\begin{tabular}{llll}
\hline & $\begin{array}{l}R^{\mathrm{ATR}} \text { of } \nu_{\text {as }} \\
\left(\mathrm{CH}_{2}\right)\end{array}$ & $f$ & $\gamma$ \\
\hline POPC & 1.18 & 0.40 & $39.2^{\circ}$ \\
POPG & 1.18 & 0.40 & $39.2^{\circ}$ \\
POPC/POPG & $1.27 \pm 0.03$ & $0.35 \pm 0.02$ & $41.2^{\circ} \pm 0.8^{\circ}$ \\
POPC/POPG and Vamp2 & $1.38 \pm 0.02$ & $0.29 \pm 0.01$ & $43.4^{\circ} \pm 0.4^{\circ}$ \\
POPC/POPG and Antp & $1.38 \pm 0.02$ & $0.29 \pm 0.01$ & $43.4^{\circ} \pm 0.4^{\circ}$ \\
POPC/POPG and Gravin & $1.55 \pm 0.03$ & $0.20 \pm 0.02$ & $46.8^{\circ} \pm 0.8^{\circ}$
\end{tabular}
${ }^{a}$ Derivations are the upper and lower limits of calculated values for
three measurements.

\subsection{Fluorescence spectra of the POPC bilayer-TrP-OMe and POPC/POPG bilayer-model peptide complexes}

Proteins that contain aromatic amino acids display a useful intrinsic fluorescence. Among the three aromatic residues, the fluorescence of Trp is most sensitive to the dielectric constant of the local environment ${ }^{49}$ because it has highest fluorescence emission intensity and highest quantum yield..$^{50}$ Therefore, the fluorescence of Trp can be used as a fluorescence probe. Interactions between the membranes and proteins can be conveniently monitored through changes in the Trp fluorescence emission wavelength and intensity.

As shown in Fig. 5A, there was an intense fluorescence emission band at $353 \mathrm{~nm}$ in the fluorescence spectrum of TrpOMe in the buffer solution, which can be assigned to the Trp residue. This band had a blue shift of about $18 \mathrm{~nm}$ when TrpOMe was mixed with the POPC bilayers, suggesting that TrpOMe bound to the POPC bilayers and the indole ring penetrated into the low dielectric region of the POPC bilayers. Blue shifts were also observed when Vamp2, Antp, and Gravin, all of which had Trp residues, were mixed with the POPC/POPG bilayers. As shown in Fig. 5B, a fluorescence emission maximum at $355 \mathrm{~nm}$ was observed for the Trp residue in Vamp2 in the buffer solution, with a blue shift to $336 \mathrm{~nm}$ when Vamp2 was added to the POPC/POPG bilayers. Similarly, the fluorescence emission peak for Antp at $351 \mathrm{~nm}$ was blue-shifted to $337 \mathrm{~nm}$ and the fluorescence emission peak at $358 \mathrm{~nm}$ for Gravin was blue-shifted to $341 \mathrm{~nm}$ when they were mixed with the POPC/POPG bilayers.

The complexes of the lipid bilayers with Trp-OMe or the three peptides were coated on the quartz surface, whereas Trp-OMe, Vamp2, Antp, and Gravin monomers were dissolved in the buffer solution and measurements were performed in quartz cuvettes. This method ensured that we were not comparing the fluorescence intensity change of the Trp residues with or without lipids. Compared with the monomers in the buffer solution, the fluorescence emission band of the Trp residues had a blue shift not only in the complexes of Trp-OMe and POPC bilayers but also in the complexes of the three Trp-containing peptides and POPC/POPG bilayers. Phe and Tyr residues did not contribute to the emission when the excitation wavelength was $295 \mathrm{~nm}$. A blue shift and increased intensity in the fluorescence emission spectra of the indole group in Trp can be observed in a completely apolar environment. ${ }^{51}$ Therefore, the blue shift in the fluorescence emission spectrum of the Trp residues in the membranes indicates that the indole ring of Trp-OMe penetrates into the low dielectric region of the POPC bilayers and the local environment surrounding the indole ring changes. Additionally, the three model peptides also bind to the POPC/POPG bilayers with the Trp residue insertion into the apolar interior of the POPC/POPG bilayers. Trp residues play a vital role in the process of peptides interacting with the membranes. Jobin et al. have reported that the number, position in the helix, and size of the hydrophobic face of the Trp residues are important for the internalization efficacy of RW9 as an efficient cell-penetrating peptide. $^{52}$ In addition, the aromatic residues in the peptides are adjacent to the positively charged basic residues on two 

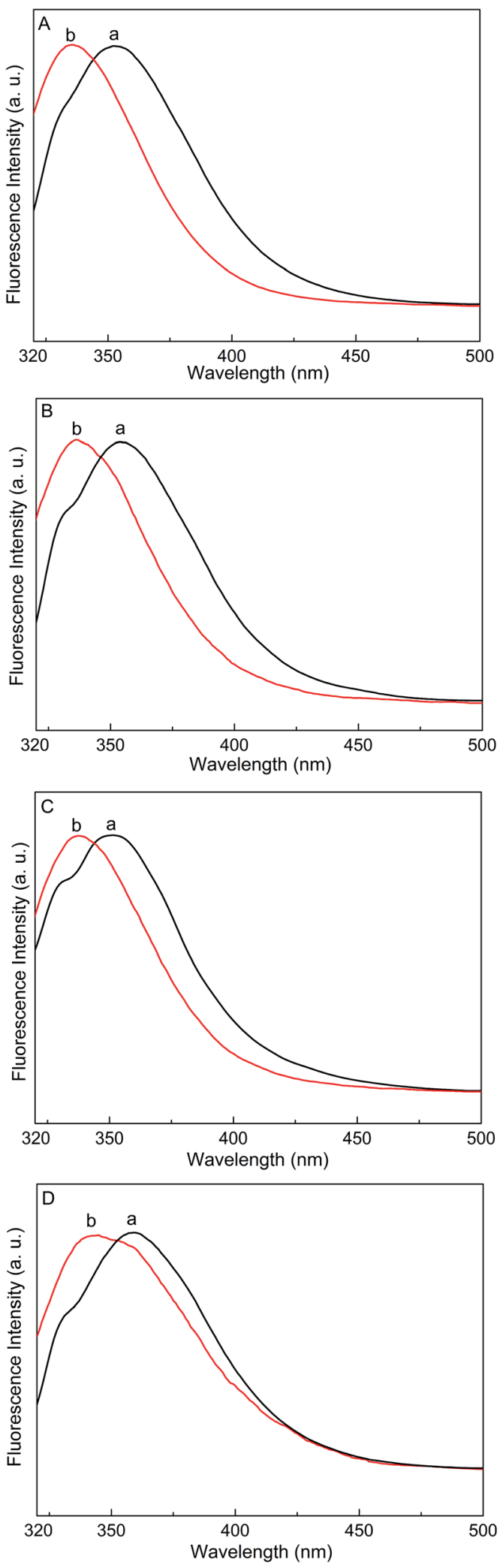

Fig. 5 Fluorescence emission spectra of the POPC bilayer-Trp-OMe and POPC/POPG bilayer-peptide complexes. (A) Fluorescence emission spectra of Trp-OMe (a) in the buffer solution and (b) in the POPC bilayers. (B) Fluorescence emission spectra of Vamp2 (a) in the buffer solution and (b) in POPC/POPG bilayers. (C) Fluorescence emission spectra of Antp (a) in the buffer solution and (b) in POPC/POPG bilayers. (D) Fluorescence emission spectra of Gravin (a) in the buffer solution and (b) in POPC/POPG bilayers. sides, and the basic residues can be pulled into the polar headgroup region; thus, it is convenient for the peptides to bind to the POPC/POPG bilayers. ${ }^{44}$ Accordingly, the aromatic residues may penetrate into the hydrophobic interior of the membrane bilayers and the peptides may bind to the POPC/POPG bilayers; thus, the conformational order of the POPC/POPG acyl chains significantly changes to different extents.

\section{Conclusions}

The acyl chain order changes of binary unsaturated lipid bilayers were investigated via polarised ATR-FTIR spectroscopy. The alterations in the frequency values of the $=\mathrm{CH}$ and $\mathrm{C}=\mathrm{C}$ stretching vibrations and dichroic properties can be used to monitor the acyl chain order of the POPC/POPG bilayers. The acyl chain order of the POPC/POPG bilayers significantly decreases as compared to that of the pure POPC or POPG bilayers. Furthermore, the chain order of the POPC/POPG bilayers is influenced by the three aromatic-residue containing peptides as well, involving in the process of the peptides interacting with the membrane bilayers, especially Gravin. In addition, the fluorescence spectroscopic measurements further supported the conclusion that the Trp of the three peptides may insert into the apolar interior region of the POPC/POPG bilayers and the peptides may bind to the POPC/POPG bilayers. Thus, the conformational order of the POPC/POPG acyl chains changes to different extents. Our findings may provide valuable information on the flexibility of the lipid bilayers with different lipid compositions and the effects of the lipid bilayers interacting with the aromatic-residue-containing peptides. It highlights the importance of aromatic residues in influencing peptide binding to the membrane and demonstrates that the stability of the membranes depends on the membrane lipid composition and the sequence, structural context, and orientation of the peptides that are bound.

\section{Abbreviations}

POPC 2-Oleoyl-1-pamlitoyl-sn-glyecro-3-phosphocholine

POPG 2-Oleoyl-1-pamlitoyl-sn-glyecro-3-glycerol

ATR- Attenuated total reflectance-Fourier transfer

FTIR infrared spectroscopy

ZnSe Zinc selenide

MARCKS Myristoylated alanine-rich C kinase substrate

FATC FAT C-terminal

PE Phosphatidylethanolamine

NR Neutron reflectivity

ESR Electron spin resonance

DPI Dual polarisation interferometry

MD Molecular dynamics

ITC Isothermal titration calorimetry

NMR Nuclear magnetic resonance

Trp-OMe Tryptophan methyl ester

HEPES 4-(2-Hydroxyethyl)-1-piperazineethanesulphonic acid 


\section{Acknowledgements}

The National Natural Science Foundation of China (grant numbers 30970657 and 21475045), Shanghai Pujiang Program (grant number 09PJ1404300), and East China Normal University (grant numbers 79003A29, 79301207, 79301411, and 41500515430-14100) are acknowledged for providing the financial support of the work.

\section{References}

1 K. Nobuhiko, I. Akihiko, I. Takehiko, N. Fumimasa, T. Shuichi and T. Kingo, Membranes, 2015, 5, 22-47.

2 M. Goto, H. Sawaguchi, N. Tamai, H. Matsuki and S. Kaneshina, Langmuir, 2010, 26, 13377-13384.

3 H. A. Scheidt, I. Haralampiev, S. Theisgen, A. Schirbel, S. Sbiera, D. Huster, M. Kroiss and P. Müller, Mol. Cell. Endocrinol., 2016, 428, 68-81.

4 M. Fidorra, T. Heimburg and H. M. Seeger, Biochim. Biophys. Acta, Biomembr., 2009, 1788, 600-607.

5 A. G. Lee, Biochim. Biophys. Acta, 2004, 1666, 62-87.

6 A. C. Johansson and E. Lindahl, J. Chem. Phys., 2009, 130, 185101.

7 A. G. Lee, Mol. BioSyst., 2005, 1, 203-212.

8 T. W. Mitchell, K. Ekroos, S. J. Blanksby, A. J. Hulbert and P. L. Else, J. Exp. Biol., 2007, 210, 3440-3450.

9 K. Kobayashi, K. Endo and H. Wada, Front. Recent Dev. Plant Sci., 2016, 7, 336.

10 J. Wang, A. Gambhir, G. Hangyás-Mihályné, D. Murray, U. Golebiewska and S. McLaughlin, J. Biol. Chem., 2002, 277, 34401-34412.

11 L. A. Sommer, M. Schaad and S. A. Dames, J. Biol. Chem., 2013, 288, 20046-20063.

12 G. Wang, B. Mishra, R. F. Epand and R. M. Epand, Biochim. Biophys. Acta, 2014, 1838, 2160-2172.

13 T. Aramaki, Y. Abe, K. Furutani, T. Katayama and T. Ueda, J. Biochem., 2015, 157, 529-537.

14 S. Serrano, A. Araujo, B. Apellaniz, S. Bryson, P. Carravilla, I. de la Arada, N. Huarte, E. Rujas, E. F. Pai, J. L. Arrondo, C. Domene, M. A. Jimenez and J. L. Nieva, J. Biol. Chem., 2014, 289, 6565-6580.

15 J. Cheng, R. Goldstein, A. Gershenson, B. Stec and M. F. Roberts, J. Biol. Chem., 2013, 288, 14863-14873.

16 G. Vitiello, G. Fragneto, A. A. Petruk, A. Falanga, S. Galdiero, A. M. D'Ursi, A. Merlino and G. D'Errico, Soft Matter, 2013, 9, 6442-6456.

17 M. R. Sanders, L. A. Clifton, R. A. Frazier and R. J. Green, Langmuir, 2016, 32, 2050-2057.

18 D. J. Hirst, T. H. Lee, M. J. Swann, S. Unabia, Y. Park, K. S. Hahm and M. I. Aguilar, Eur. Biophys. J., 2011, 40, 503-514.

19 T. D. Romo, L. A. Bradney, D. V. Greathouse and A. Grossfield, Biochim. Biophys. Acta, Biomembr., 2011, 1808, 2019-2030.

20 K. Witte, B. E. Olausson, A. Walrant, I. D. Alves and A. Vogel, Biochim. Biophys. Acta, 2013, 1828, 824-833.
21 R. N. A. H. Lewis and R. N. McElhaney, Biochim. Biophys. Acta, Biomembr., 2013, 1828, 2347-2358.

22 G. Güler, R. M. Gärtner, C. Ziegler and W. Mäntele, J. Biol. Chem., 2016, 291, 4295-4307.

23 S. Ergun, P. Demir, T. Uzbay and F. Severcan, Biochim. Biophys. Acta, Biomembr., 2014, 1838, 2798-2806.

24 Z. D. Schultz and I. W. Levin, Annu. Rev. Anal. Chem., 2011, 4, 343-366.

25 M. Arczewska, D. M. Kamiński, E. Górecka, D. Pociecha, E. Rój, A. Sławińska-Brych and M. Gagoś, Biochim. Biophys. Acta, Biomembr., 2013, 1828, 213-222.

26 B. Peng, X.-Y. Ding, C. Sun, W. Liu, J. Z. H. Zhang and X. Zhao, RSC Adv., 2016, 6, 45569-45577.

27 E. Sikorska, M. Dawgul, K. Greber, E. Iłowska, A. Pogorzelska and W. Kamysz, Biochim. Biophys. Acta, Biomembr., 2014, 1838, 2625-2634.

28 M. G. K. Benesch, R. N. A. H. Lewis and R. N. McElhaney, Biochim. Biophys. Acta, Biomembr., 2016, 1858, 168-180.

29 F. Korkmaz, S. Köster, Ö. Yildiz and W. Mäntele, Biochemistry, 2008, 47, 12126-12134.

30 R. Ishiguro, N. Kimura and S. Takahashi, Biochemistry, 1993, 32, 9792-9797.

31 M. Pritsker, J. Rucker, T. L. Hoffman, R. W. Doms and Y. Shai, Biochemistry, 1999, 38, 11359-11371.

32 A. Menikh, M. T. Saleh, J. Gariépy and J. M. Boggs, Biochemistry, 1997, 36, 15865-15872.

33 I. T. Arkin, W. P. Russ, M. Lebendiker and S. Schuldiner, Biochemistry, 1996, 35, 7233-7238.

34 S. O. Smith, R. Jonas, M. Braiman and B. J. Bormann, Biochemistry, 1994, 33, 6334-6341.

35 L. K. Tamm and S. A. Tatulian, Biochemistry, 1993, 32, 77207726.

36 O. K. Gasymov, A. R. Abduragimov, T. N. Yusifov and B. J. Glasgow, Protein Sci., 2000, 9, 325-331.

37 A. Blume, Curr. Opin. Colloid Interface Sci., 1996, 1, 64-77.

38 H. L. Casal and H. H. Mantsch, Biochim. Biophys. Acta, Rev. Biomembr., 1984, 779, 381-401.

39 L. Janosi and A. A. Gorfe, J. Chem. Theory Comput., 2010, 6, 3267-3273.

40 S. A. Tatulian, Methods Mol. Biol., 2013, 974, 177-218.

41 C. Vigano, L. Manciu, F. Buyse, E. Goormaghtigh and J. M. Ruysschaert, Pept. Sci., 2000, 55, 373-380.

42 F.-X. Ding, H. Xie, B. Arshava, J. M. Becker and F. Naider, Biochemistry, 2001, 40, 8945-8954.

43 B. Ding and Z. Chen, J. Phys. Chem. B, 2012, 116, 25452552.

44 A. Gambhir, G. Hangyas-Mihalyne, I. Zaitseva, D. S. Cafiso, J. Y. Wang, D. Murray, S. N. Pentyala, S. O. Smith and S. McLaughlin, Biophys. J., 2004, 86, 2188-2207.

45 A. M. Bouchet, F. Lairion, J. M. Ruysschaert and M. F. Lensink, Chem. Phys. Lipids, 2012, 165, 89-96.

46 M. Hoernke, C. Schwieger, A. Kerth and A. Blume, Biochim. Biophys. Acta, Biomembr., 2012, 1818, 1663-1672.

47 B. Christiaens, S. Symoens, S. Vanderheyden, Y. Engelborghs, A. Joliot, A. Prochiantz, J. Vandekerckhove, M. Rosseneu and B. Vanloo, Eur. J. Biochem., 2002, 269, 2918-2926. 
48 B. Christiaens, J. Grooten, M. Reusens, A. Joliot, M. Goethals, J. Vandekerckhove, A. Prochiantz and M. Rosseneu, Eur. J. Biochem., 2004, 271, 1187-1197.

49 D. Williams, J. Vicogne, I. Zaitseva, S. McLaughlin and J. E. Pessin, Mol. Biol. Cell, 2009, 20, 4910-4919.

50 C. A. Kraft, J. L. Garrido, L. Leiva-Vega and G. Romero, Sci. Signaling, 2009, 2, p14.
51 J. R. Lakowicz, Principles of Fluorescence Spectroscopy, Springer, New York, 3rd edn, 2006.

52 M.-L. Jobin, M. Blanchet, S. Henry, S. Chaignepain, C. Manigand, S. Castano, S. Lecomte, F. Burlina, S. Sagan and I. D. Alves, Biochim. Biophys. Acta, Biomembr., 2015, 1848, 593-602. 